Article

\title{
Optical Fiber Pumped High Repetition Rate and High Power Nd:YVO 4 Picosecond Regenerative Amplifier
}

\author{
Zhen-Ao Bai ${ }^{1, *}$, Zhong-Wei Fan ${ }^{1}$, Zhen-Xu Bai ${ }^{2, *}$, Fu-Qiang Lian ${ }^{1}$, Zhi-Jun Kang ${ }^{1}$ \\ and Wei-Ran Lin ${ }^{1}$
}

1 Academy of Opto-Electronics, Chinese Academy of Sciences, Beijing 100049, China; E-Mails: zhongwei_fan@sina.com (Z.-W.F.); aoefiberlaser@126.com (F.-Q.L.); kzjun1221@126.com (Z.-J.K.); linweiran@aoe.ac.cn (W.-R.L.)

2 National Key Laboratory of Science and Technology on Tunable Laser, Harbin Institute of Technology, Harbin 150001, China

* Authors to whom correspondence should be addressed; E-Mails: baizhenao@hotmail.com (Z.-A.B.); baizhenxu@hotmail.com (Z.-X.B.); Tel.: +86-10-8217-8661 (Z.-A.B.).

Academic Editor: Malte C. Kaluza

Received: 7 July 2015 / Accepted: 4 August 2015 / Published: 13 August 2015

\begin{abstract}
We report a stable optical fiber pumped $\mathrm{Nd}: \mathrm{YVO}_{4}$ all solid state regenerative amplifier with all fiber picosecond laser as seed source. $888 \mathrm{~nm}$ Yb optical fiber lasers was chosen as pump source to reduce quantum defect for improved thermal performance. At the repetition rate of $99.6 \mathrm{kHz}$, maximum power of $19.63 \mathrm{~W}$ with 36 ps pulse duration were achieved when seeded by a $150 \mathrm{~mW}$ picosecond oscillator. The wavelength delivered was $1064.07 \mathrm{~nm}$ with spectral width of $0.14 \mathrm{~nm}$.
\end{abstract}

Keywords: picosecond laser; all solid state; high repetition rate; regenerative amplifier

\section{Introduction}

Picosecond lasers with compact structure, lightweight and high stability are in demand for a number of applications, such as laser satellite ranging (SLR), precision machining, biology, spectroscopy, and military science [1-5]. All fiber picosecond laser has the characteristics of miniaturization and lightweight, however, the peak power of all fiber laser operating in the picosecond regime is limited to the nano-Joule level due to the strong nonlinear effect in high peak power operation. In order to generate 
stable high peak power regenerative amplifier output, a good way is to combine the fiber picosecond seed source with all solid state regenerative amplification technology [6-8].

In order to obtain high peak power regenerative amplified output at high repetition rate, we need to improve the injected pumping power as well as the pump absorption efficiency. At present, $808 \mathrm{~nm}$ wavelength is the most widely used pumping source of $\mathrm{Nd}: \mathrm{YVO}_{4}$ crystal for the highest absorption peaks around $810 \mathrm{~nm}$. However, due to the poor thermal and mechanical properties of $\mathrm{Nd}: \mathrm{YVO}_{4}$, a large temperature gradient will be generated inside the crystal at high power pumping. For example, the heat capacity and conductivity of $\mathrm{Nd}$ :YAG are $0.59 \mathrm{~J} /(\mathrm{g} \cdot \mathrm{K})$ and $0.13 \mathrm{~W} /(\mathrm{cm} \cdot \mathrm{K})$, respectively; however, as for $\mathrm{Nd}: \mathrm{YVO}_{4}$ are corresponding to $0.79 \mathrm{~J} /(\mathrm{g} \cdot \mathrm{K})$ and $0.06 \mathrm{~W} /(\mathrm{cm} \cdot \mathrm{K})$. High local stress can even cause the damage of crystal, which limits the increase of pumping power [9-12]. In addition, the $\mathrm{Nd}: \mathrm{YVO}_{4}$ absorption cross section for $\pi$ and $\sigma$ polarizations differ by a factor of 3.7 at $808 \mathrm{~nm}$. That is to say, for an $\boldsymbol{a}$-axis cutting $\mathrm{Nd}: \mathrm{YVO}_{4}$ crystal, the optical absorption coefficient in $808 \mathrm{~nm}$ has great difference along the two orthogonal axis. Although $\mathrm{Nd}: \mathrm{YVO}_{4}$ crystal has lower absorption efficiency with $888 \mathrm{~nm}$ $(0.15 / \mathrm{mm}$ along $\boldsymbol{a}$ - $\& \boldsymbol{c}$-axis) than that of $808 \mathrm{~nm}(1.0 / \mathrm{mm}$ and $3.7 / \mathrm{mm}$, respectively), we can also improve absorption efficiency by increasing the crystal length or Nd-doping concentration. The increase of the crystal length means the increase of the heat dissipation area and the decrease of the thermal effect. Compared with $0.8 \mathrm{~nm}$ absorption band in $808 \mathrm{~nm}$ pumping, $\mathrm{Nd}: \mathrm{YVO}_{4}$ absorption band in $888 \mathrm{~nm}$ pumping is $3 \mathrm{~nm}$ which is not sensitive to the fluctuation of the wavelength jitter. Therefore, $808 \mathrm{~nm}$ pumped $\mathrm{Nd}: \mathrm{YVO}_{4}$ regenerative amplifier is able to achieve stable output in the case of high pumping power at high repetition rate.

In this paper, we develop a high power and high repetition rate all solid state $\mathrm{Nd}: \mathrm{YVO}_{4}$ picosecond regenerative amplifier by using all optical fiber picosecond laser as seed source and $888 \mathrm{~nm}$ laser as pumping source. The regenerative amplifier is operating at $99.6 \mathrm{kHz}$ delivering up to $19.63 \mathrm{~W}$ output power with pulse duration $39 \mathrm{ps}$, central wavelength $1064.07 \mathrm{~nm}$ and spectral width $0.14 \mathrm{~nm}$. To our best knowledge, this is the highest power has been generated from an optical fiber end pumped all solid-state picosecond regenerative amplifier at $\sim 100 \mathrm{kHz}$ repetition rates.

\section{Experimental Section}

Design scheme of the regenerative amplifier is shown in Figure 1. It consists of a fiber seed, an optical splitter, an optical isolator and a regenerative amplifier. After passing through the beam collimator and optical isolation, the seed pulses are derived into two parts. One part enter into the photodetector (PD) as the clock signal, the other part inject into the regenerative amplifier.

The seed we chose is a commercial mode-locked all fiber oscillator that produces $150 \mathrm{~mW}$ at repetition rate $46.8 \mathrm{MHz}$. The pulse duration of the seed pulses is $20 \mathrm{ps}$ with a central wavelength of $1064.06 \mathrm{~nm}$ and spectral width $0.2 \mathrm{~nm}$. The optical splitter is composed of a half wave plate and a polarized beam splitter (PBS). By adjusting the half wave plate, we can control the seed power injected into the regenerative amplifier and $\mathrm{PD}$. After adjustment, $100 \mathrm{~mW}$ power is injected into the regenerative amplifier, and the rest of the $50 \mathrm{~mW}$ entered into the PD. Optical isolator consists of a polarizer, a half wave plate and a Faraday (FR) that just allows the light transmission in the direction of injection. Regenerative amplifier is composed of a polarizer, a quarter wave plate, an electro-optic Q-switch, a gain medium, a pumping source and many total reflection mirrors. The total length of the cavity is about $2200 \mathrm{~mm}$. 
Inside the regenerative amplifier, the $\mathrm{Nd}: \mathrm{YVO}_{4}$ crystal is end pumped by $888 \mathrm{~nm}$ continuous wave (CW) laser. The 0.5 -at. $\% \mathrm{Nd}: \mathrm{YVO}_{4}$ crystal has dimensions of $3 \times 3 \times 20 \mathrm{~mm}^{3}$ with $2^{\circ}$ wedge angle in one end. To improve the cooling efficiency, $\mathrm{Nd}: \mathrm{YVO}_{4}$ crystal is welded on heat sink that cooled in both $\boldsymbol{x}$ - and $\boldsymbol{y}$ - directions. Used as a pulse picker, Pockels cell (PC) consists of two BBO crystals with single crystal size of $3 \times 3 \times 20 \mathrm{~mm}^{3}$. PC is operated with quarter-wave voltage. The rise and fall times of the $\mathrm{PC}$ driver are about $6 \mathrm{~ns}$. The chirp of $\mathrm{Nd}: \mathrm{YVO}_{4}$ and BBO crystals is about 1-2 ps. M1 and M5 are two plano-concave high reflectance (HR) mirrors with $\mathrm{R}=-2000 \mathrm{~mm}$. M2 and M4 are two plane-convex HR mirrors with $\mathrm{R}=1500 \mathrm{~mm}$ to compensate the thermal focal length of the medium. $\mathrm{M} 3$ is a $45^{\circ}$ plane mirror coated with antireflection at $888 \mathrm{~nm}$ and highly reflective at $1064 \mathrm{~nm}$. The pump source is an $888 \mathrm{~nm}$ semiconductor fiber coupling module with maximum output power $100 \mathrm{~W}$. Output fiber core diameter is $400 \mu \mathrm{m}$, and the pump beam is coupled into the crystal by a 1:4 coupling lens telescope.

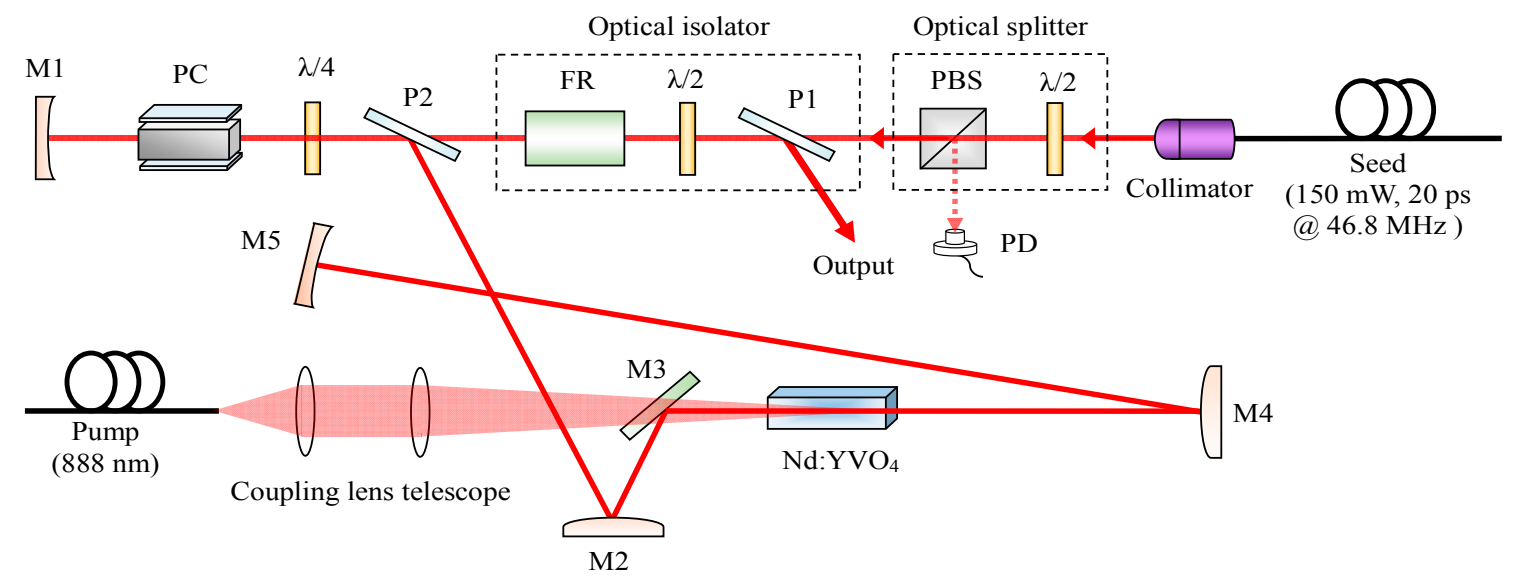

Figure 1. Design scheme of the fiber laser pumped regenerative amplifier.

\section{Results and Discussion}

When the absorbed pump power is $73.8 \mathrm{~W}$, maximum regenerative amplified output power $19.6 \mathrm{~W}$ was generated at $99.6 \mathrm{kHz}$ in center wavelength of $1064.06 \mathrm{~nm}$ and spectral width of $0.14 \mathrm{~nm}$. Here, the un-picked seed pulses are amplified to $150 \mathrm{~mW}$ when switching off the high voltage of the PC. The pump source spectrum is shifted to the long wavelength with the increase of the power. As shown in Figure 2, the central wavelength of the pump source is $887.66 \mathrm{~nm}$ with spectral width of $1.84 \mathrm{~nm}$ when operating at $73.8 \mathrm{~W}$.

The curve of output power varies with pump absorbed power is shown in Figure 3. It can be seen from the figure that the output power increases gradually with the increase of the pump power. The highest output power achieved is $19.6 \mathrm{~W}$ while the absorbed power of medium is $73.8 \mathrm{~W}$.

As shown in Figure 4, different regenerative amplification time corresponding to different output pulse energy. The output energy is relatively stable, about $196 \mu \mathrm{J}$, when the regeneration amplification time ranges from $180 \mathrm{~ns}$ to $225 \mathrm{~ns}$.

The stable pulse train, unstable multi-period pulse train trace and a single pulse profile of the regenerative amplified output are shown in Figure $5(\mathrm{a}-\mathrm{c})$, respectively. Stable pulse train is obtained with the regenerative amplification time of $195 \mathrm{~ns}$ at repetition rate of $99.6 \mathrm{kHz}$, as shown in Figure 5 (a). As the regenerative amplification time continues to increase, along with the phenomenon of multi-period, 
the output pulse train becomes no longer stable with repetition rate jitter from about $20 \mathrm{kHz}$ to $50 \mathrm{kHz}$, as illustrated in Figure 5 (b).

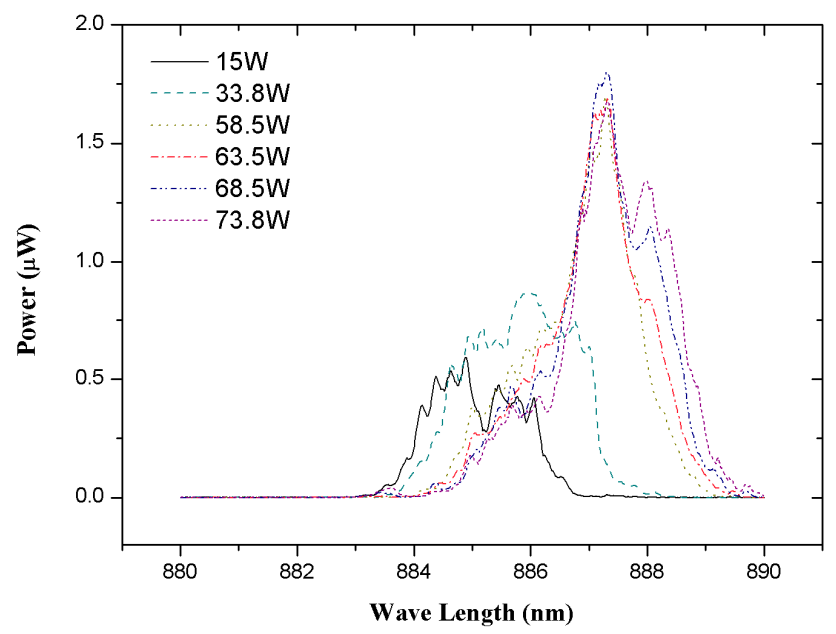

Figure 2. The spectral intensity distribution as a function of absorbed pump power.

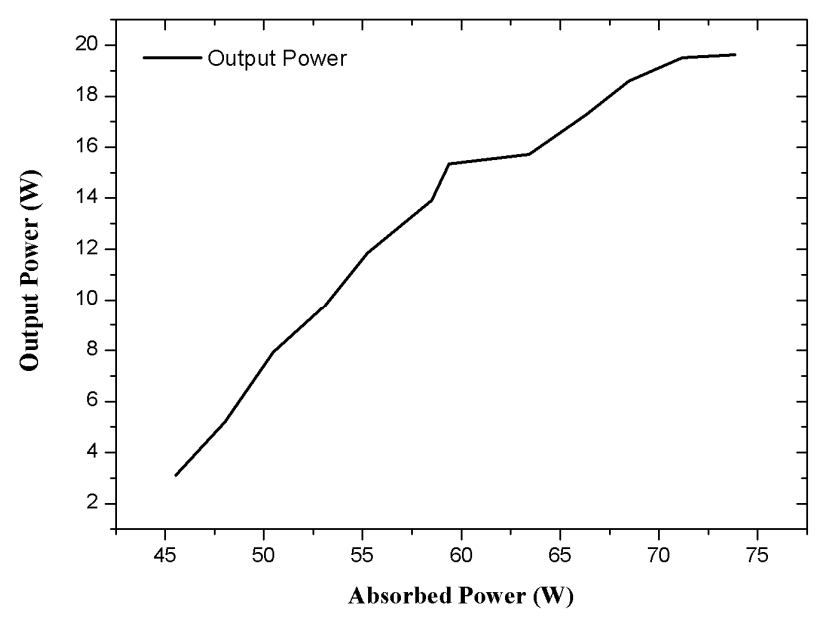

Figure 3. The output power as a function of absorbed power in operation.

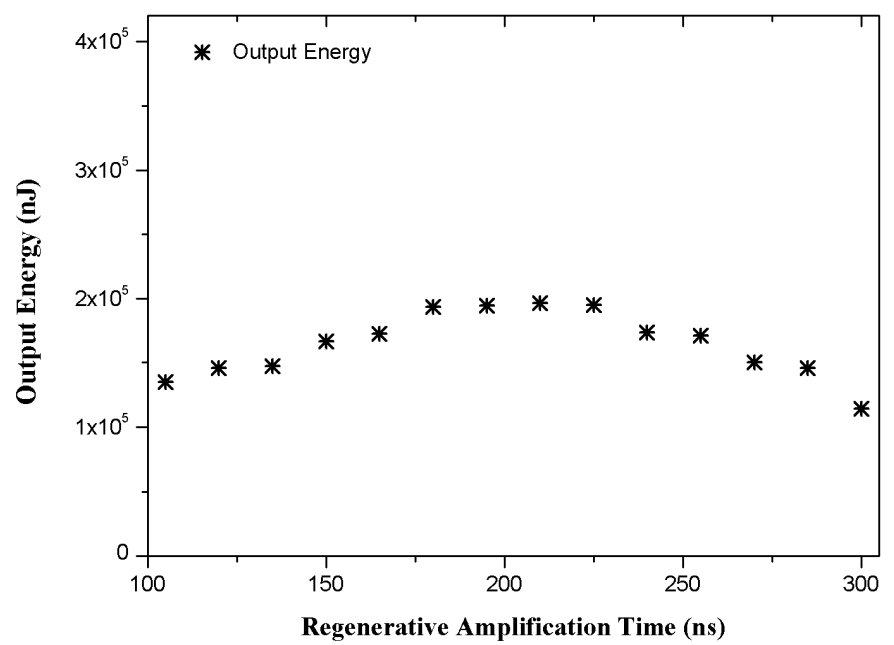

Figure 4. The output energy as a function of regeneration amplification time in operation. 

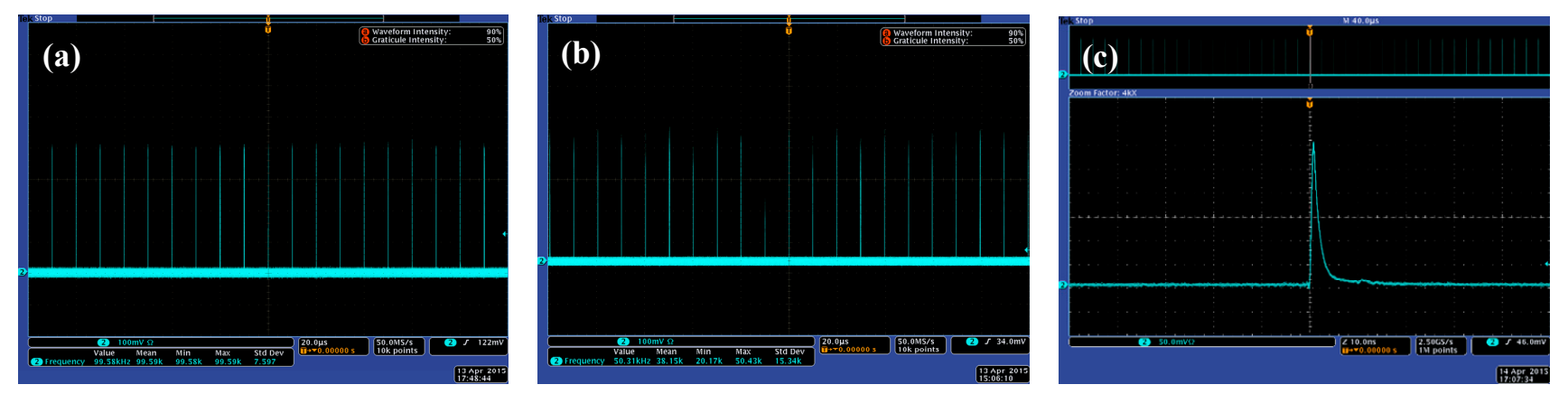

Figure 5. Oscilloscope traces of (a) the pulse train at $195 \mathrm{~ns}$ regeneration amplification time; (b) the pulse train of unstable multi-period phenomenon; (c) a single pulse shape at $195 \mathrm{~ns}$ regeneration amplification time.

After regenerative regeneration, spectrum distribution of the injected seed is slightly changed, as depicted in Figure 6. Solid line is the spectral intensity distribution curve of seed source with center wavelength $1064.06 \mathrm{~nm}$ and the spectral width $0.2 \mathrm{~nm}$. Broken line is corresponding to the regenerative amplified output spectral intensity with center wavelength $1064.07 \mathrm{~nm}$ and spectral width $0.14 \mathrm{~nm}$. Results shows that gain narrowing tends to compress the optical spectrum.

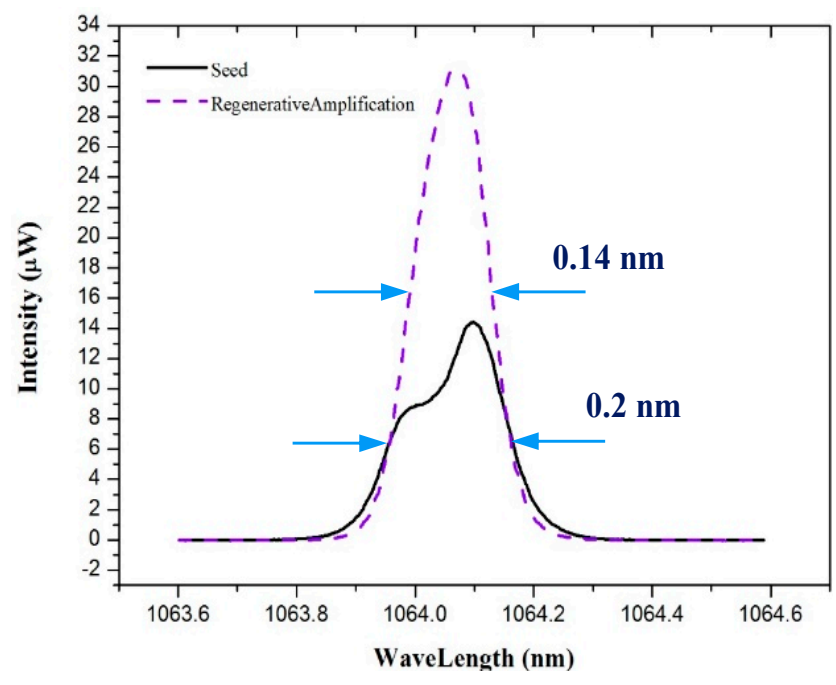

Figure 6. Spectral intensity distribution of the injected seed (solid line) and regenerative amplified output (broken line).

The autocorrelation signal shows that the output pulse duration is $36 \mathrm{ps,}$, as illustrated in Figure 7. Compared with the pulse duration 20 ps of the injected picosecond seed source, the output pulse duration is broadened widely. According to the Fourier transform limited pulse, time-bandwidth product (TBWP) must be equal to or greater than the constant $\kappa[13,14]$ :

$$
\Delta t \cdot \Delta v \geq \kappa
$$

where $\Delta t$ is the full width at half maximum (FWHM) of pulse envelope intensity, $\Delta v$ is the FWHM of Fourier transform spectroscopy. The TBWP for a Gauss pulse is $\kappa=2 \ln 2 / \pi=0.441$. The TBWP of the fiber picosecond seed source is 1.06 , and the regenerative amplified output pulse TBWP is 1.34. 
In our experiment, on the one hand, the reason for pulse duration being broader is gain narrowing, and the other is chirp phenomenon.

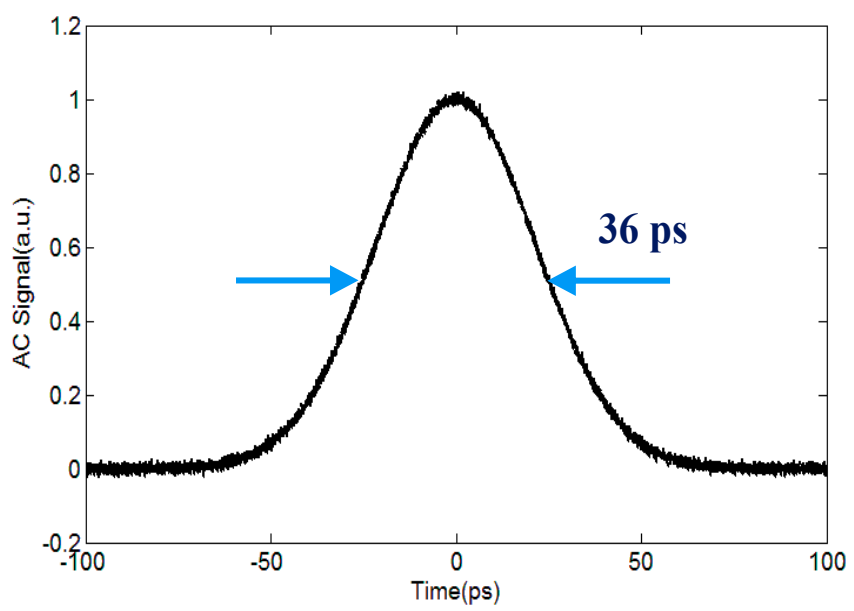

Figure 7. Autocorrelation signal of pulse output from the regenerative amplifier.

Figure 8 represents the beam characteristics of the output, which indicates nearly diffraction limited beam quality. The result giving a beam quality of $M_{x}^{2}=1.20, M_{y}^{2}=1.19$ in both directions perpendicular to the axis of propagation. The beam diameter in $\boldsymbol{x}$ direction is $1.5 \mathrm{~mm}$ and $\boldsymbol{y}$ direction is $1.3 \mathrm{~mm}$. The slight deviation of the two direction of the spot is caused by the $2^{\circ}$ wedge angle of the crystal.
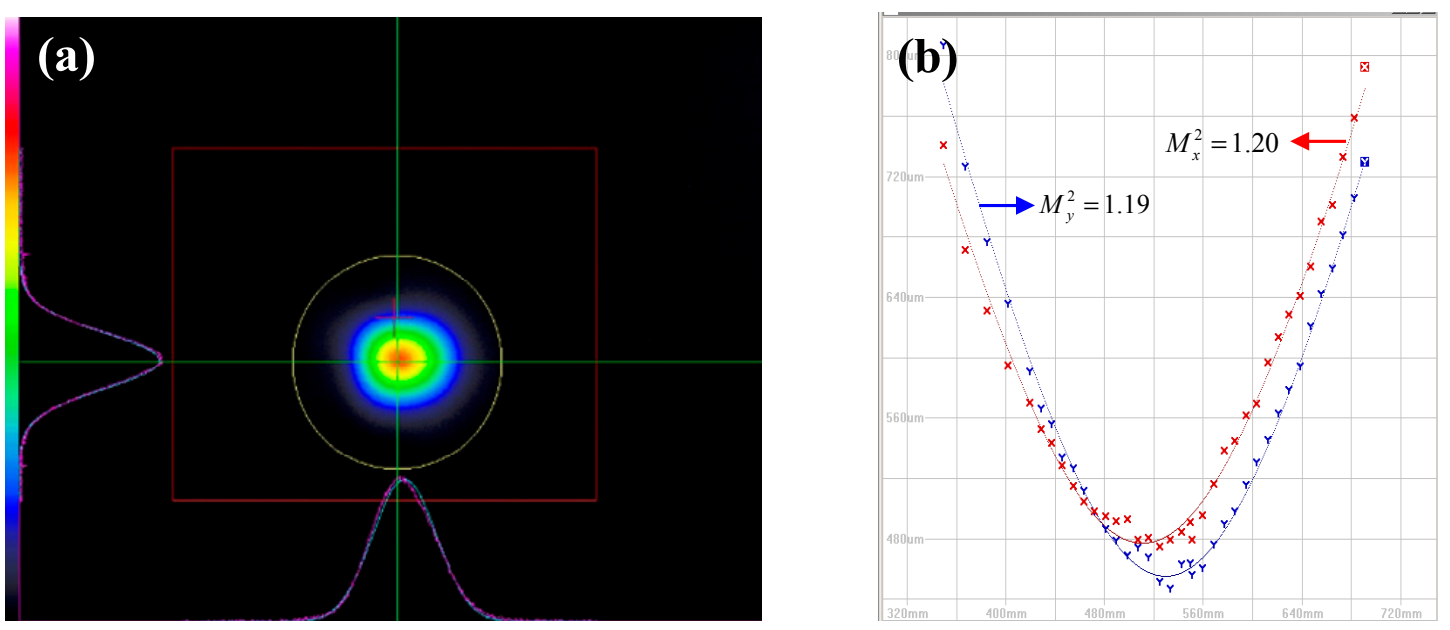

Figure 8. Measured beam characteristics of (a) profile of the output with $\boldsymbol{x}$ direction $1.5 \mathrm{~mm}$ and $\boldsymbol{y}$ direction $1.3 \mathrm{~mm} ;(\mathbf{b}) \mathrm{M}^{2}$ with $M_{x}^{2}=1.20$ and $M_{y}^{2}=1.19$.

\section{Conclusions}

In summary, we have demonstrated an optical fiber end pumped high average power and high repetition rate picosecond regenerative amplifier. To reduce the radial temperature gradient of $\mathrm{Nd}: \mathrm{YVO}_{4}$ crystal, $888 \mathrm{~nm}$ pumping source is adopted. The influence of different regeneration time on the output waveform, as well as the spectrum distribution of the injected seed and regenerative amplified output are studied. We achieved $19.63 \mathrm{~W}$ regenerative amplified output power at $99.6 \mathrm{kHz}$ for $73.8 \mathrm{~W}$ pump 
power when the injected seed power is $150 \mathrm{~mW}$. The output pulse duration was $36 \mathrm{ps}$ in central wavelength $1064.07 \mathrm{~nm}$ and spectral width $0.14 \mathrm{~nm}$. Close to the diffraction limit output is obtained with beam quality factors of $M_{x}^{2}=1.20$ and $M_{y}^{2}=1.19$. This work offers a new approach to generate high power regenerative amplifier, which is able to deliver sub-millijoule energies regenerative amplified pulses at up to $\sim 100 \mathrm{kHz}$ repetition rates.

\section{Acknowledgments}

This work is supported by the Development of High Power Nanosecond Laser \& Precision Detecting Instrument Foundation (grant No. ZDYZ2013-2).

\section{Author Contributions}

Zhenao Bai made the study design and performed the experiment, as well as the manuscript writing. Zhongwei Fan provided the ideas and facilities. Zhenxu Bai participated in research plan development and revised the manuscript. Fuqiang Lian, Zhijun Kang and Weiran Lin contributed to the data analysis and results discussion. All authors have contributed to the manuscript and have approved the final version.

\section{Conflicts of Interest}

The authors declare no conflict of interest.

\section{References}

1. Gerhard, C.; Druon, F.; Blandin, P.; Hanna, M.; Balembois, F.; Georges, P.; Falconz, F. Efficient versatile-repetition-rate picosecond source for material processing applications. Appl. Opt. 2003, 47, 967-974.

2. Yang, F.M.; Chen, W.Z.; Zhang, Z.P.; Chen, J.P.; Hu, J.F.; Li, X.; Prochazka, I.; Hamal, K. Satellite laser ranging experiment with sub-centimeter single-shot ranging precision at Shanghai Observatory. Sci. China Ser. G 2003, 46, 84-88.

3. Cerny, P.; Zverev, P.G.; Jelinkova, H.; Basiev, T.T. Efficient Raman shifting of picosecond pulses using $\mathrm{BaWO}_{4}$ crystal. Opt. Commun. 2000, 177, 397-404.

4. Roy, S.; Gord, J.R.; Patnaik, A.K. Recent advances in coherent anti-Stokes Raman scattering spectroscopy: Fundamental developments and applications in reacting flows. Prog. Energy Combust. Sci. 2010, 36, 280-306.

5. Spiro, A.; Lowe, M.; Pasmanik, G. Drilling rate of five metals with picosecond laser pulses at 355, 532, and 1064 nm. Appl. Phys. A 2012, 107, 801-808.

6. Kim, J.W.; Shen, D.Y.; Sahu, J.L.; Clarkson, W.A. Fiber-Laser-Pumped Er:YAG Lasers. IEEE J. Sel. Top. Quantum Electron. 2009, 15, 361-371.

7. Chang, C.L.; Krogen, P.; Hong, K.H.; Zapata, L.E.; Moses, J.; Calendron, A.L.; Liang, H.K.; Lai, C.J.; Stein, G.J.; Keathely, P.D.; et al. High-energy, kHz, picosecond hybrid Yb-doped chirped-pulse amplifier. Opt. Express 2015, 23, 10132-10144. 
8. Lian, F.Q.; Fan, Z.W.; Bai, Z.A.; Yu, J.; Lin, W.R.; Zhang, X.L.; Liu, D.; Zhao, T.Z. A Nd:YAG regenerative amplifier seeded by $1064 \mathrm{~nm}$ picosecond fiber. Acta. Phys. Sin. 2014, 63, doi:10.1117/12.874203.

9. McDonagh, L.; Wallenstein, R.; Nebel, A. $111 \mathrm{~W}, 110 \mathrm{MHz}$ repetition-rate, passively mode-locked $\mathrm{TEM}_{00} \mathrm{Nd}: \mathrm{YVO}_{4}$ master oscillator power amplifier pumped at $888 \mathrm{~nm}$. Opt. Lett. 2007, 32, 1259-1261.

10. Lührmann, M.; Harth, F.; Theobald, C.; Ulm, T.; Knappe, R.; Nebel, A.; Klehr, A.; Erbert, G.; Lhuillier, J.A. High average power $\mathrm{Nd}: \mathrm{YVO}_{4}$ regenerative amplifier seeded by a gain switched diode laser. SPIE Proc. 2011, 7912, doi:10.1117/12.874203.

11. Yang, C.; Chen, M.; Li, G. High-Pulse-Energy Passively Mode-Locked Nd:YVO 4 Laser without Spatial Hole Burning Effect. Opt. Rev. 2014, 21, 256-260.

12. Siebold, M.; Hornung, M.; Hein, J.; Paunescu, G.; Sauerbrey, R.; Bergmann, T.; Hollemann, G. A high-average-power diode-pumped $\mathrm{Nd}: \mathrm{YVO}_{4}$ regenerative laser amplifier for picosecond-pulses. Appl. Phys. B 2004, 78, 287-290.

13. Bai, Z.A.; Bai, Z.X.; Yang, C.; Chen, L.Y.; Chen, M.; Li, G. High pulse energy, high repetition picosecond chirped-multi-pulse regenerative amplifier laser. Opt. Laser Technol. 2013, 46, 25-28.

14. Wada, K.; Cho, Y. Improved expression for the time-bandwidth product of picosecond optical pulses from gain-switched semiconductor lasers. Opt. Lett. 1994, 19, 1633-1635.

(C) 2015 by the authors; licensee MDPI, Basel, Switzerland. This article is an open access article distributed under the terms and conditions of the Creative Commons Attribution license (http://creativecommons.org/licenses/by/4.0/). 